Relations industrielles

Industrial Relations

\title{
Administration industrielle et générale, par Henri Fayol, édition présentée par P. Morin, Paris, Dunod, 1979, 156 pp.
}

\section{Dimitri Weiss}

Volume 35, numéro 2, 1980

URI : https://id.erudit.org/iderudit/029079ar

DOI : https://doi.org/10.7202/029079ar

Aller au sommaire du numéro

Éditeur(s)

Département des relations industrielles de l'Université Laval

ISSN

0034-379X (imprimé)

1703-8138 (numérique)

Découvrir la revue

Citer ce compte rendu

Weiss, D. (1980). Compte rendu de [Administration industrielle et générale, par Henri Fayol, édition présentée par P. Morin, Paris, Dunod, 1979, 156 pp.] Relations industrielles / Industrial Relations, 35(2), 350-351.

https://doi.org/10.7202/029079ar

Tous droits réservés @ C Département des relations industrielles de l'Université Laval, 1980
Ce document est protégé par la loi sur le droit d'auteur. L’utilisation des services d'Érudit (y compris la reproduction) est assujettie à sa politique d'utilisation que vous pouvez consulter en ligne.

https://apropos.erudit.org/fr/usagers/politique-dutilisation/ 
tiens pour un livre de référence, particulièrement en Europe latine, sur la médiation des pouvoirs publics dans les conflits collectifs du travail: La mediazione dei publici poteri nei conflitti collettive di lavoro (Bologna, Il Mulino, 1972). L'auteur, Bruno Veneziani, dont j'ai déjà eu l'occasion de parler dans ces colonnes à propos de deux autres volumes collectifs parus en 1978 (voir Relations industrielles, vol. 34, no. 1, 1979, pp. 205-206), est également le signataire d'une contribution sur le même sujet au 26e volume de l'Encyclopédie du Droit: "Mediazione nelle controversie collettive di laboro" (Enciclopedia del diritto, Milano, Giuffrè Editore, 1976).

\section{Dimitri WEISS}

Institut d'Administration des Entreprises, Paris

Les nouvelles formes d'organisation du travail, vol. 2, Genève, Bureau international du travail, 1979, $161 \mathrm{pp}$.

Dans un premier volume (Les nouvelles formes d'organisation du travail, 1979), le Bureau international du travail a déjà publié cinq monographies sur les nouvelles formes d'organisation du travail au Danemark, en Norvège et en Suède, en République fédérale d'Allemagne, en France, au Royaume-Uni et aux États-Unis. Dans ce deuxième volume, le BIT s'intéresse à des expériences tentées en Italie, en Inde, en République démocratique allemande et en URSS.

Bien que l'avant-propos comprenne un avertissement concernant le caractère disparate des analyses regroupées dans ce second volume, le lecteur est néanmoins assuré d'y trouver une trame, un commun dénominateur. En effet, tous les auteurs manifestent d'une façon ou d'une autre une préoccupation envers des considérations théoriques: fondements des systèmes d'organisation du travail, évolution du marché de l'emploi, répercussions du progrès technique, principes d'organisation etc. De plus, pour chaque pays, on s'attarde à décrire le système d'organisation du travail en vigueur ainsi que la démarche jugée la plus appropriée pour con- tribuer à son amélioration. Enfin, dans tous les cas, des exemples concrets sont présentés.

Mais là s'arrêtent les points communs. Certaines monographies ont été rédigées par des chercheurs indépendants tandis que d'autres l'ont été par des spécialistes à l'emploi d'organismes of ficiels. Les opinions émises à l'égard de l'organisation du travail varient largement et se situent de façon différente par rapport au système socio-économique des pays concernés. En regroupant ces monographies, l'objectif du BIT n'était pas de prendre position sur les opinions exprimées mais de montrer «comment les chercheurs ou les spécialistes des organismes of ficiels envisagent le problème dans différents pays». Au lecteur de juger. Le choix des expériences est varié et comprend aussi bien des groupes semi-autonomes dans la métallurgie italienne que l'application du système scientifique d'organisation du travail dans l'industrie textile soviétique.

Cette publication du BIT comprend également un article de Anthony Hopwood consacré à l'évaluation de l'intérêt économique des nouvelles formes d'organisation du travail. À la suite de son analyse, l'auteur conclut que les normes visant à améliorer la qualité de la vie au travail, bien qu'elles soient parfois coûteuses, «peuvent avoir une incidence positive sur les résultats économiques généraux des entreprises». À partir d'exemples, il discute des facteurs agissant sur les résultats économiques des mesures d'amélioration de la qualité de la vie au travail. Cependant, ce type d'évaluation n'est pas sans poser de délicats problèmes de mesure au point d'exiger souvent une remise en cause de la façon traditionnelle d'envisager les performances et les résultats de l'entreprise.

Alain VINET

Université Laval

Administration industrielle et générale, par Henri Fayol, édition présentée par P. Morin, Paris, Dunod, 1979, 156 pp.

Les spécialistes et observateurs des relations industrielles sont familiarisés avec ce 
qu'on appelle, depuis un temps déjà, le Taylorisme, et ce particulièrement depuis que les problèmes de l'organisation du travail ont cessé d'être excentriques pour occuper la place qui est actuellement la leur, au coeur même de notre domaine d'études et d'action.

Que ne pourrait pas en dire autant du fayolisme, terme peu usité, que Gérard Dion n'a pas hésité, heureusement, d'introduire dans son Dictionnaire canadien des relations du travail, en le référant, naturellement au premier.

Et Pierre Morin, dans la présentation de l'Administration industrielle et générale, publié, à l'origine, dans le Bulletin de la Société de l'industrie minérale de 1916, et deux ans plus tard par le (ré) éditeur d'aujourd'hui, Dunod, explique bien comment -et pourquoi - Fayol, le Français, trop méconnu en France même, et surtout, malgré sa fameuse séquence "prévoir, organiser, commander, coordonner, contrôler», a vécu dans l'ombre de son contemporain américain Taylor (qu'il cite et commente), trop célèbre peut-être, ce qui ne manque de surprendre.

Car Fayol "revisité» surprend par sa modernité, au moins dans une vue managériale dont nous voyons bien, en ce début de décennie, l'intérêt revigoré. Peut-il en être de même pour le relationniste (industriel)?

Il y a des éléments non-négligeables qui méritent d'être relus, en tenant compte du contexte historique, et dont on ne peut s'empêcher de constater la fortune à travers divers courants qui se sont succédés au cours des dernières décennies.

Fayol en fut, à sa façon, un précurseur, à l'époque peu compris et peu suivi. Et Morin va jusqu'à supposer que ses idées sur le social auraient pu, si elles avaient été suivies, changer la physionomie du monde industriel français.

Pierre Morin était tout désigné pour préfacer cette édition. Consultant d'entreprises et formateur expérimenté, auteur d'un Développement des organisations (Dunod, 2e édition, 1976) et plus récemment, co-auteur et éditeur de Commander demain (Dunod, 1978), il est le type même, et rare, du psychosociologue pour lequel la gestion n'a plus de secrets.

\section{Dimitri WEISS}

Institut d'Administration des Entreprises, Paris

The Health Care Business. International Evidence on Private Versus Public Health Care Systems, by Ake Blomqvist, Vancouver, B.C., The Fraser Institute, 1979, 185 pp.

Le livre de l'économiste Blomqvist est publié par le Fraser Institute. Comme tel, il s'inscrit fidèlement dans la ligne de pensée de cet organisme dont l'objectif avoué est de porter à l'attention du public les vertus de l'économie de marché.

C'est un livre qui m'a plu à plus d'un titre. D'abord, le texte est bien structuré et les titres fréquents permettent de dégager facilement les idées maîtresses. De plus, l'auteur n'hésite pas à livrer des opinions personnelles qui contribuent à animer le texte.

Le premier chapitre est consacré à la formulation du problème. Le lecteur, déjà familier avec le livre de Migué et Bélanger «Le prix de la santé», se sentira en terrain connu. Blomqvist y reprend sensiblement les mêmes critiques à l'endroit de notre système de distribution des soins de santé basé sur le principe d'assurance intégrale et la rémunération à l'acte des médecins.

Étant donné que le patient n'est pas vraiment un consommateur souverain, le médecin se voit placer en situation de conflit d'intérêts en ce sens que son propre revenu est directement relié aux décisions qu'il prend dans le cadre de la relation avec son patient. Sous un régime, comme le nôtre, d'assurance intégrale où par surcroît la prime est payée de façon indirecte via les taxes et les impôts, l'arbitrage prix-qualité est à toute fin pratique absent de la relation médecin-patient. On peut alors s'attendre à une poussée inflationniste. 